\title{
Grundzüge einer Löß- und Bodenstratigraphie am Niederrhein
}

\author{
Von Karl Brunnacker, Köln
}

Mit 2 Abbildungen

\begin{abstract}
Zusatin e nfassung. Die Deckschichten-Profile der Rheinterrassen unterhalb Kölns zeigen prinzipiell den gleichen Aufbau, wie er aus dem nördlichen Alpenvorland bekannt ist. Auf der Krefelder Mittelterrasse tritt Löß einer nachfolgenden Kaltzeit auf (z. B. Holzheim). Die Untere Mittelterrasse trägt Deckschichten von zwei jüngeren Kaltzeiten (Giesenkirchen). Auf der Jüngeren Hauptterrasse liegt eine reich gegliederte Abfolge, welche den Deckschichten über den sogenannten Riesenböden von Regensburg (K. BrunnaCKeR 1964a) verwandt ist - also vier Kaltzeiten vertritt, von denen die älteste weiter unterteilt ist (Wegberg).

$\mathrm{Sum}$ mary. Profiles of the covering sediments from the Rhine terraces north of Cologne, can be compared with ones of similar structure found in the northern Alpine foreland. On the Krefeld Middle-terrace is a glacial period, represented by a loess-layer (e. g. Holzheim). The Lower Middle-terrace has covering sediments attributable to two younger glacial periods (Giesenkirchen). On the Younger Main-terrace of the Rhine is a very detailed formation, which profile is related to the covering sediments over the so-called "Riesenböden“ from Regensburg K. BRUNNACKER, 1964a). The formation from the Younger Main-terrace would then comprise four glacial periods, with the oldest glacial having further subdivisions (Wegberg).

Immer wieder wird betont, daß periglaziale Deckschichten auf eiszeitlichen Terrassen samt den damit verbundenen fossilen Böden hinsichtlich ihrer stratigraphischen Bedeutung von nur begrenzter Aussagekraft sind, weil sie lediglich über das Mindestalter des Liegenden Angaben zu erbringen vermögen. Trotzdem lohnt es sich, das Alter auf diese Weise zusätzlich einzuengen; denn die anderen zur Verfügung stehenden Hilfsmittel sind zumeist noch viel spärlicher vertreten und von oft noch problematischerer Natur. Voraussetzung ist allerdings eine sinnvolle Interpretation der Deckschichten und der damit im Verband befindlichen Paläoböden.
\end{abstract}

Trotz zahlreicher moderner genetischer und stratigraphischer Untersuchungen über die Deckschichten und vor allem die Paläoböden am Mittel- und Niederrhein durch E. Mückenhausen (1954, 1955, 1959), J. Frechen \& E. A. Rosauer (1959), H. Remy (1959, 1960), H. Remy \& W. PaAs (1959), W. PaAs (1961) und E. H. Müller (1959) sind gerade hinsichtlich der Beziehungen zu den Terrassen manche Fragen offen geblieben. Die Gründe dafür sind nur z. T. im Bereich der Deckschichten zu suchen. Sie beruhen auch im Mangel an einer eindeutigen Terrassen-Korrelation als Ausgangsbasis - zumindest insoweit als es sich um die Mittelterrassen handelt. Aus diesem Grunde beschränken sich die folgenden Ausführungen allein auf die weitere Umgebung Kölns. Es ist das Verdienst von W. PAas (1961), durch systematische Bearbeitung der Paläoböden und Deckschichten am Niederrhein eine Diskussionsgrundlage geschaffen zu haben. Seiner zusammenfassenden Abb. 32 nach ist es allerdings schwierig, klare Beziehungen zu den Terrassen des Rheintales zu finden. Darüber hinaus ist es sowohl unmöglich, eine allgemeine Verbindung zur sonstigen Lößgliederung Mitteleuropas herzustellen, wie eine Übereinstimmung mit den speziellen Verhältnissen im Alpenvorland zu erkennen.

Auf einige Detailfragen dieses Raumes, so die Fazies des würmeiszeitlichen Lösses und dessen Feinstratigraphie, ferner die Lößfazies und die Paläoböden im allgemeinen und die Deckschichten auf einer Unterstufe der Oberen Mittelterrasse sowie auf eine (keinesfalls komplette) Deckschichtenfolge der Jüngeren Hauptterrasse, wurde inzwischen eingegangen (K. Brunnacker 1966b, Fl. Heller \& K. Brunnacker 1966). Einige weitere Profile, die für unsere Fragestellung interessant sind, werden nachfolgend besprochen. 
Die Deckschichten der Terrassen bestehen aus Löß, Lößlehm und einem als Fleckenlehm bezeichneten Lößlehm, sowie dem noch stärker veränderten Staublehm. Löß und normaler Lößlehm treten bevorzugt in der letzten Kaltzeit, Fleckenlehm in der vorletzten und Staublehm in der drittletzten Kaltzeit auf. In noch älteren Kaltzeiten kommen Hochflutlehme hinzu, die hier zweckmäßigerweise in die Deckschichten einbezogen werden. Die zwischengeschalteten, ebenfalls z. T. schon behandelten, interglazialen Böden sind als Parabraunerden und Pseudogleye, bei Hochflutabsätzen auch als Auenböden und Gleye entwickelt. Hinsichtlich der Sedimentausbildung und der Paläoböden liegen damit im Vergleich zu anderen Gebieten entsprechende Bauelemente vor.

\section{a. Niederterrasse}

Der würmeiszeitlichen Niederterrasse fehlen naturgemäß Deckschichten einer nachfolgenden, durch eine interglaziale Warmzeit davon abgesetzten Kaltzeit. Die Auflage von Schwemmlöß, Hochflutlehm usw. bildet vielmehr den Abschluß der fluviatilen Aufschüttungsphase der letzten Kaltzeit. Darauf braucht an dieser Stelle nicht näher eingegangen zu werden.

\section{b. Krefelder Mittelterrasse}

Der Aufbau würmeiszeitlicher Periglazialprofile am Niederrhein wurde bereits an anderer Stelle erörtert: Getrennt durch den Boden des letzten Interglazials liegt eine solche würmeiszeitliche Deckschicht, keinesfalls aber mehr, auf der Krefelder Mittelterrasse. Diese Deckschicht ist wohl durch interstadiale Tundrenböden weiter aufgeteilt; ihr ermangeln aber auf wärmere Bedingungen weisende Böden kräftigerer Entwicklung. Die recht komplizierte Abfolge auf der Krefelder Mittelterrasse, wie sie W. PAAs (1961) vorführt, läßt sich zumindest für den Kölner Raum stark vereinfachen:

Würmeiszeitlicher Löß

$\mathrm{R} / \mathrm{W}$-interglazialer Boden

Hochflutlehm, mit kryoturbaten Störungen zu Beginn seiner Ablagerung

Schotter der Krefelder Mittelterrasse.

Ein entsprechender Aufbau findet sich z. B. auch auf der rißeiszeitlichen Hochterrasse im Donautal bei Straubing. Der dort die Aufschotterung abschließende Schwemmlöß, zugeführt aus einem Seitentälchen, ist von Frostspalten durchsetzt; darüber folgt der interglaziale Boden und dann der Würmlöß. Es ist damit auch gar nicht nötig, gemäß H. REMY (1963) die Hochterrasse des Alpenvorlandes für eine Parallelisierung entsprechend zurecht zu rücken.

Da weitere einschlägige Profile gelegentlich behandelt werden, kann als Beispiel das Profil von Holzheim genügen (Abb. 1).

Profil Holzh e i m (vgl. W. PaAs 1961)

Tiefe in $\mathrm{cm}$

0- 50 Brauner, brockiger, kräftiger, feinsandiger Lehm, graufleckig (darüber rd. $30 \mathrm{~cm}$ abgeräumt)

-100 brauner, brockiger, kräftiger, feinsandiger Lehm, graufleckig, Verfahlungen an Schwundrissen

-140 brauner, feinsandiger, schwach kräftiger Lehm, schwach körnig, mit Eisenkonkretionen $(3 \mathrm{~mm} \phi)$

-155 braun und graugelb schlierig gebänderter, kräftiger, feinsandiger Lehm

-175 hellgrünlichgrauer, stark feinsandiger Lehm, an Obergrenze Anreicherung von Eisenkonkretionen $(1 \mathrm{~cm} \phi)$, allmählicher Ubergang nach unten in:

-225 rötlichbrauner, bröckeliger, feinsandiger, kräftiger Lehm, stark graufleckig, Verfahlungen an Schwundrissen, Tontapeten auf Bodenkörpern

-265 brauner, feinsandiger, kräftiger Lehm, brockig bis plattig, graufleckig, Tontapeten auf Bodenkörpern

Kryoturbationshorizont

-285 anlehmiger Sand, hellgraublau und rostbraun gefleckt

-335 graugelber Sand mit bis $5 \mathrm{~cm}$ dicken Rostbändern (Krefelder Mittelterrasse). 

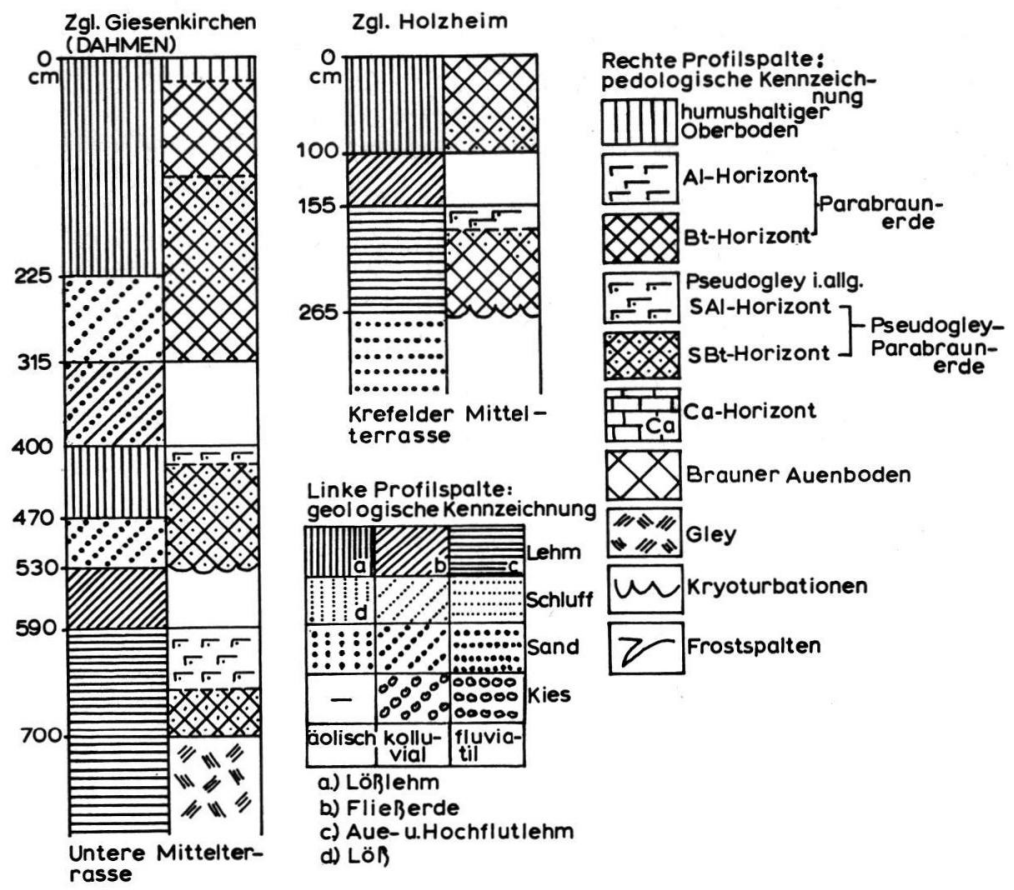

Abb. 1. Deckschichtenprofile auf der Krefelder und Unteren Mittelterrasse (Profildarstellung entsprechend den Vorschlägen der Subkommission für Löß- und Bodenstratigraphie der INQUA vgl. J. FinK 1965).

De utung :

\begin{tabular}{|c|c|c|}
\hline $0-100$ & Pseudovergleyte Parabraunerde & heutiger Boden \\
\hline \multirow{2}{*}{$0-155$} & Lößlehm & \\
\hline & Fließerde & letzte Kaltzeit \\
\hline $155-265$ & Pseudogley-Parabraunerde & letzte Warmzeit \\
\hline $155-335$ & $\begin{array}{l}\text { Hochflutlehm } \\
\text { Kryoturbationen } \\
\text { Terrassenrand }\end{array}$ & vorletzte Kaltzeit \\
\hline
\end{tabular}

c. Untere Mittelterrasse

Relativ vollständige Deckschichtenprofile über der Unteren Mittelterrasse sind anscheinend sehr selten. Das Profil von Giesenkirchen (Abb. 1) stellt W. PaAs (1961) aus nicht ersichtlichen Gründen auf die Krefelder Mittelterrasse. Deren Außenrand verläuft jedoch rund $0,5 \mathrm{~km}$ nördlich davon, bei einer Niveaudifferenz der heutigen, aus Löß gebildeten Landoberfläche von rd. $5 \mathrm{~m}$. Zweifellos liegt dieses Profil auf der Unteren Mittelterrasse.

P r of il Gi e s e n k i r h e n (Zgl. Dahmen; vgl. W. PaAs 1961)

Tiefe in $\mathrm{cm}$

0- 25 Humushaltiger, dunkelgrauer, feinsandiger Lehm

-125 rötlichbrauner, kräftiger, feinsandiger Lehm, brockig

-225 brauner, kräftiger, feinsandiger Lehm, mit sandigen Schlieren und Bändern, graufleckig, längs Klüfte Verfahlungen (Horizont keilt seitlich aus)

-315 hellbrauner, lehmiger Sand, rostbraun gebändert

-330 graugelber Sand, unten kiesig 
Tiefe in $\mathrm{cm}$

-390 hellgraugelber, schwach sandiger, feinsandiger Lehm

-400 graugelber, schluffiger Lehm

-420 hellgrauer, schluffiger Lehm

-470 rötlichbrauner, kräftiger, schluffiger Lehm, brockig bis bröckelig, Tonhäutchen auf Bodenkörpern, stark gelbgraufleckig

-530 hellbrauner, lehmiger Sand, unten kiesig, längs Schwundrisse grau verfahlt, unten bis $5 \mathrm{~cm}$ dicke Rostbänder

Kryoturbationshorizont

- 550 graubrauner, feinsandiger Lehm

-580 hellbrauner, kräftiger Lehm

-590 hellgrauer, schluffiger Lehm

-650 hellgraugelber, schluffiger Lehm, stark graufleckig

--700 brauner, kräftiger Lehm, bröckelig bis brockig, graufleckig

-720 grauer, kräftiger Lehm, mit Rostbändern und stark rostfleckig

-800 grauer, kräftiger Lehm

D e u t u g :

\begin{tabular}{|c|c|c|}
\hline \multicolumn{2}{|c|}{$0-225(-315)$ Pseudovergleyte Parabraunerde } & heutiger Boden \\
\hline $0-400$ & $\begin{array}{l}\text { Lößlehm } \\
\text { Lößlehm im Wechsel mit Schwemmlehm und Sand }\end{array}$ & letzte Kaltzeit \\
\hline
\end{tabular}

\begin{tabular}{|c|c|c|}
\hline \multicolumn{2}{|c|}{ 400-470(-530) Pseudogley-Parabraunerde } & \multirow[t]{2}{*}{ letzte Warmzeit } \\
\hline $400-530$ & Lößlehm & \\
\hline & $\begin{array}{l}\text { Schwemmsand } \\
\text { Kryoturbationen }\end{array}$ & vorletzte Kaltzeit \\
\hline $530-700$ & Pseudogley-Parabraunerde & vorletzte Warmzeit \\
\hline $530-800$ & Hochflutlehm (schwemmlößartig) & drittletzte Kaltzeit \\
\hline
\end{tabular}

Sandige Terrassensedimente werden in diesem Profil durch eine schwemmlößartige Decke überlagert, deren Material aus einem benachbarten Seitental zugeführt wurde. Daraus entstand ein interglazialer Boden mit sekundärer Pseudovergleyung. Darüber liegen verschwemmter kiesiger Sand, dann Lößlehm, die als Deckschicht zur nachfolgenden Kaltzeit gehören. Eine pseudovergleyte Parabraunerde bildet deren hangenden Abschluß. Weiterer kiesiger Sand und Lößlehm einer zweiten Deckschicht tragen den rezenten Boden.

Dem Profil nach muß die unterlagernde Terrasse um ene Etage älter sein als die Krefelder Mittelterrasse des Kölner Raumes. Entsprechend den Verhältnissen an der Donau ist die Zuordnung dieser Terrasse in die drittletzte, also die Mindeleiszeit möglich. Freilich werden damit Fragen hinsichtlich der Einstufung des Drenthe-Eisvorstoßes an den Niederrhein aufgeworfen. Deren Erörterung würde jedoch vom eigentlichen Thema zu weit wegführen, zumal es sich fragt, ob das Problem durch die Aufteilung der SaaleEiszeit in Drenthe und Warthe im üblichen Sinne überhaupt zutreffend formuliert ist.

d. Rinnenschotter und Obere Mittelterrassen

Die älteren Terrassen am Niederrhein werden wie folgt eingeteilt:

Hauptterrassen

Obere Mittelterrassen

\section{Untere Mittelterrasse \\ Krefelder Schichten \\ Rinnenschotter}

Der Mittleren Mittelterrasse am Mittelrhein soll am Niederrhein der Rinnenschotter entsprechen. $\mathrm{Ob}$ allerdings dieser Rinnenschotter Repräsentant einer ganzen Eiszeit ist, bleibt nach den Untersuchungen von E. KempF (1966) fraglicher denn je zuvor. Die Un- 
tergliederung der Oberen Mittelterrassen unterhalb Kölns wurde bereits kurz erörtert (Fl. Heller \& K. Brunnacker 1966). Hinsichtlich der Deckschichten ist in diesem Niveau mit bereits derart großen Lücken zu rechnen, daß die Auflagerungen nur noch ausnahmsweise eine Hilfe bei der Datierung von Terrassen bieten. Immerhin ist es möglich, daß auf der jüngeren dieser Oberen Mittelterrassen bei Niederaussem die Deckschichten von drei Eiszeiten und, bei etwas vereinfachter Interpretation der Angaben von E. MücKeNHAUSEN (1955), auf einer älteren Oberen Mittelterrasse eine noch reichere Abfolge liegen.

Ein anderer Gesichtspunkt bietet sich durch das eventuelle Vorkommen von Riesenböden an (K. BRUNNACKER 1964a u. b). Ein solcher, heute allerdings durch Abbau verschwundener Pseudogley wird als Basisboden der Deckschichten von E. MückenHausen (1955) beschrieben. Bei konsequenter Weiterführung der Deckschichtengliederung, aber auch der Terrassenfolge, gehört die jüngere Obere Mittelterrasse in die viertletzte Kaltzeit, während die ältere Obere Mittelterrasse davon möglicherweise durch einen Riesenboden abgesetzt ist.

e. Haupterrassen

Von den Hauptterrassen ist vorläufig nur die Jüngere Hauptterrasse von Interesse. Innerhalb derselben können bezüglich der Böden und Deckschichten zwei Typen unterschieden werden. Einmal finden sich auf der Jüngeren Hauptterrasse weiter verbreitet sehr ausgeprägte Böden von $10 \mathrm{~m}$ und mehr Mächtigkeit. Hier sind die Deckschichten bemerkenswert kümmerlich entwickelt. Daneben gibt es Stellen mit stärker aufgeteilten Deckschichten. An solchen Lokalitäten fehlt nach den bisherigen Beobachtungen ein den Schotter abschließender Boden, oder es findet sich nur eine sehr schwache Verwitterungszone (z. B. in Rheindahlen, K. BRunNacker 1966). Es ist denkbar, daß sich in den mächtigen Böden die Wirkungen mehrerer Warmzeiten summieren. Offen bleibt vorläufig auch, ob sich darin regionale Gesetzmäßigkeiten abzeichnen.

Das vollständige Deckschichtenprofil der Jüngeren Hauptterrasse am Niederrhein ist in der Ziegelei Simons bei Wegberg aufgeschlossen. Es liegt im Erosionsschatten einer bis $13 \mathrm{~m}$ hohen, durch eine Verwerfung bedingten Geländestufe (vgl. L. Ahorner 1962). Nächst der Verwerfung hat sich auf der abgesunkenen Scholle ein Tälchen entwickelt, dessen Füllung, deren Deckschichten und Paläoböden folgenden Aufbau zeigen (Abb. 2):

Profil W e g b e r g (vgl. Simons; vgl. W. PaAs 1961)

Profil A:

Tiefe in $\mathrm{cm}$

0- 15 Humushaltiger, dunkelgraubrauner, bröckeliger, feinsandiger Lehm

- 30 schwach humushaltiger, braungrauer, feinsandiger Lehm, plattig, stark rostfleckig an den Absonderungsflächen

- 65 rostbrauner, brockiger, kräftiger, feinsandiger Lehm, graufleckig

-145 lehmiger Sand, mit einzelnen Kiesschnüren, hellgelbgrau und rostbraun gebändert (5-20 cm Dicke der Bänder)

Kryoturbationen

-185 feinsandiger Lehm, mit cm-dicken Sandschmitzen, hellgrau und braun gebändert, schwach rostfleckig

-225 brauner, kräftiger, feinsandiger Lehm, bröckelig bis brockig, Tonhäutchen auf den Bodenkörpern

-300 hellbrauner, feinsandiger Lehm, mit graugelben Flecken $(1 \mathrm{~cm} \phi)$

-400 hellgraubrauner, feinsandiger, schluffiger Lehm, geschichtet, mit cm-dicken Sandlinsen

-405 brauner, kiesiger Sand

-430 hellgelbbrauner, feinsandiger Lehm, stark graufleckig, schwach rostfleckig, mit Eisenkonkretionen $(5 \mathrm{~mm} \phi)$

-470 hellgraubrauner, schwach sandiger, feinsandiger Lehm, grau- und rostfleckig, mit zahlreichen Eisenkonkretionen $(5 \mathrm{~mm} \phi)$

-540 rostbrauner, kiesiger, sandiger Lehm bis feinsandiger Lehm, bröckelig bis brockig, Tonhäutchen auf Bodenkörpern, stark grau- und rostfleckig, Verfahlungen längs der Schwundrisse 
Profil A

Profil B

Profil C

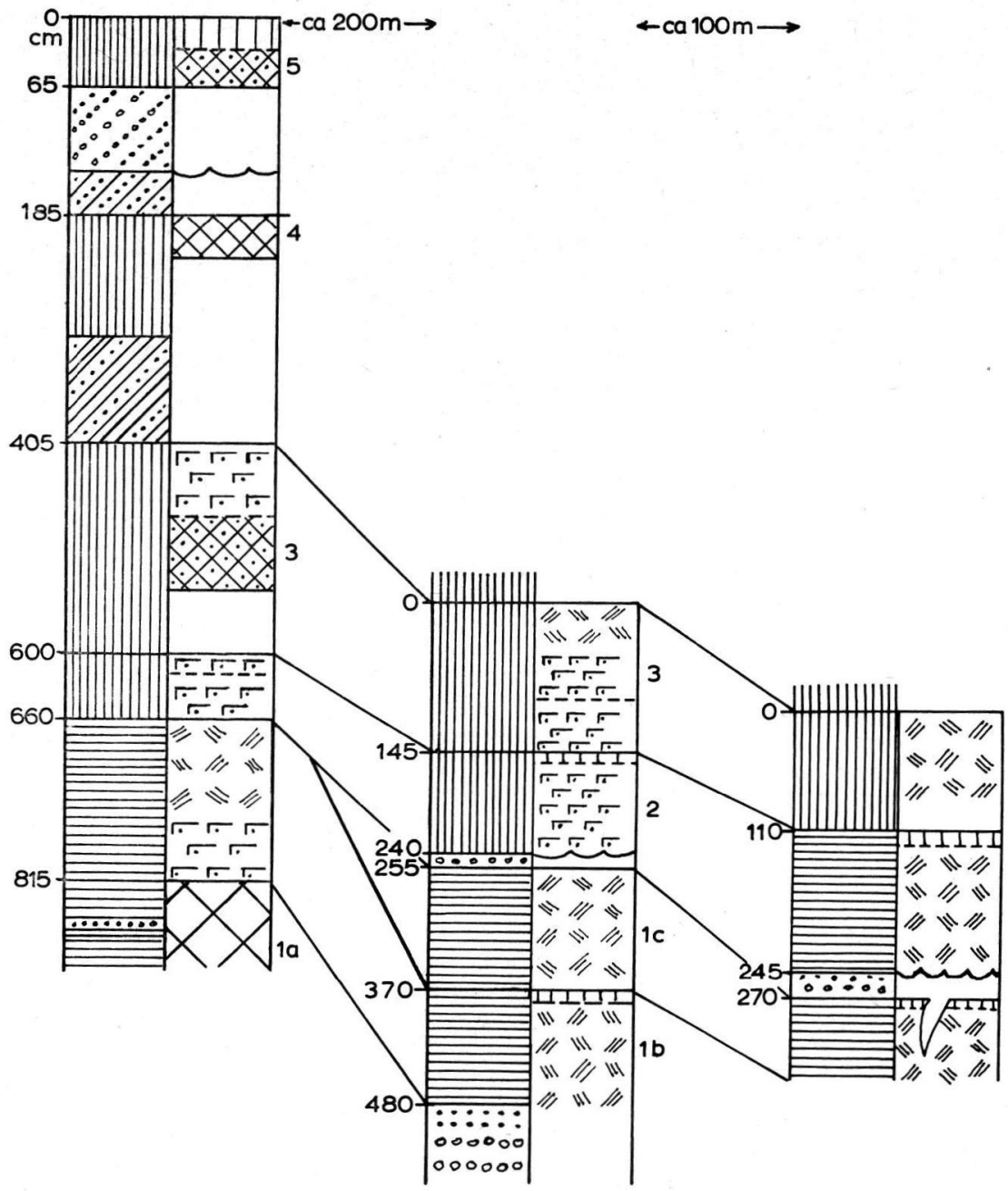

Abb. 2. Deckschichtenprofile in der Zgl. Simons in Wegberg auf der Jüngeren Hauptterrasse Kryoturbationen

Tiefe in $\mathrm{cm}$

-600 feinsandiger, schwach kräftiger Lehm, dichtgelagert, hellgrau und braun marmoriert, mit Eisenkonkretionen $(2 \mathrm{~mm} \phi)$

-620 hellgelbgrauer, feinsandiger Lehm, stark graufleckig, schwach rostfleckig, zahlreiche Eisenkonkretionen $(5 \mathrm{~mm} \phi)$

-660 hellgraubrauner, feinsandiger Lehm, stark graufleckig, rostfleckig, nächst Oberfläche sehr stark rostfleckig, plattig absondernd

-695 hellgelbgrauer, feinsandiger Lehm, stark graufleckig, Roströhren $(1 \mathrm{~cm} \phi)$ und $\mathrm{cm}$-dicke Rostbänder

-735 hellgelbgrauer, feinsandiger Lehm, stark graufleckig, schwach rostfleckig

-815 schwach sandiger Lehm, hellgrau und rostbraun großfleckig marmoriert, oben zahlreiche Eisenkonkretionen $(5 \mathrm{~mm} \varnothing)$, plattige Absonderung, längs der Oberfläche stark rostfleckig

-900 rostbrauner, kiesiger, schwach lehmiger Sand, schwach graufleckig. 
Profil B:

Im Hangenden $5 \mathrm{~m}$ Lößlehm nicht aufgenommen, darunter:

Tiefe in $\mathrm{cm}$ :

$0-45$ Hellbraungrauer, feinsandiger Lehm, mit Roströhren $(2 \mathrm{~cm} \phi)$

- 95 hellgrauer, stark lehmiger Feinsand

-145 hellgraubrauner, stark feinsandiger Lehm, stark grau- und rostfleckig, plattige Abson-

derungen mit Rostabscheidungen an deren Grenzflächen

-150 hellbraungrauer, stark feinsandiger Lehm

-240 hellgraugelber, kräftiger, feinsandiger Lehm, stark grau- und rostfleckig, plattige Absonderung, an der Oberfläche Rostausscheidung

Kryoturbationen

-255 rostbrauner, kiesiger, lehmiger Sand, hellgraubraun gebändert, rostfleckig

-300 hellbraungrauer, feinsandiger, kräftiger Lehm, stark rostfleckig, plattig absondernd,

Roströhren $(1 \mathrm{~cm} \phi)$

-370 hellbraungrauer, feinsandiger Lehm, Roströhren nach unten abnehmend

-410 hellgrauer, feinsandiger Lehm, oben stark rostfleckig und mit violettstichigen dunkel-

grauen, schwach humushaltigen Schlieren

-480 hellgelbgrauer, stark feinsandiger Lehm, rostfleckig

-520 hellgrauer Sand, rostfleckig

-550 graugelber, sandiger Kies, mit Rostbändern.

Profil C:

Im Hangenden $4 \mathrm{~m}$ Lößlehm nicht aufgenommen, darunter:

Tiefe in $\mathrm{cm}$

0- 60 Hellbraungrauer, kräftiger Lehm, plattig absondernd, stark rostfleckig, mit Roströhren $(1 \mathrm{~cm} \phi)$

- 90 hellgrauer, stark feinsandiger Lehm

-110 grauer, feinsandiger Lehm, sehr schwach humushaltig

-125 schwach humushaltiger, dunkelviolettgrauer, feinsandiger Lehm, schwach rostfleckig

-195 hellgelbgrauer, feinsandiger Lehm, rostfleckig, oben sehr schwach humushaltig

-245 hellgelbgrauer, feinsandiger Lehm, plattig absondernd, stark rostfleckig, mit Roströhren $(1 \mathrm{~cm} \phi)$

Kryoturbationen

-270 hellgrauer, kiesiger, lehmiger Sand mit cm-dicken Rostbändern, als Füllung von Frostspalten in die darunter liegenden Horizonte hineingreifend

-275 sehr schwach humushaltiger, violettstichig-grauer, kiesiger, lehmiger Sand

-300 hellgrauer, feinsandiger Lehm, plattig absondernd, stark rostfleckig

-350 hellgelbgrauer, feinsandiger Lehm, mit Roströhren $(1 \mathrm{~cm} \phi)$

Die zeitlichen und räumlichen Unterschiede im Profilaufbau sind durch die von Profil A nach C zunehmende Annäherung an das genannte Tälchen bedingt.

Deutung :

Profil A

Profil B

Profil C

Abschnitt

0- 65 Pseudovergleyte

Parabraunerde

$\begin{array}{lll}\text { 0-185 } & \begin{array}{l}\text { Lößlehm } \\ \text { Schwemmsand }\end{array} & \begin{array}{l}\text { nicht } \\ \text { aufgenommen }\end{array} \\ & \text { und Kies } & \end{array}$

\begin{tabular}{ll}
\hline $185-225$ & Parabraunerde \\
\hdashline $185-405$ & $\begin{array}{l}\text { Lößlehm } \\
\text { Fließerde }\end{array}$
\end{tabular}

\begin{tabular}{|c|c|c|c|c|c|c|}
\hline $405-540$ & $\begin{array}{l}\text { Pseudogley- } \\
\text { Parabraunerde }\end{array}$ & $\begin{array}{l}0-45 \\
0-145\end{array}$ & $\begin{array}{l}\text { sek. Gley } \\
\text { Pseudogley }\end{array}$ & $0-90$ & Gley & 3 \\
\hline $405-600$ & Lößlehm & $0-145$ & Lößlehm & $0-110$ & Lößlehm & \\
\hline $600-660$ & Pseudogley & $145-260$ & Pseudogley & $110-195$ & Naßgley & 2 \\
\hline $600-660$ & Lößlehm & $145-255$ & $\begin{array}{l}\text { Lößlehm } \\
\text { Kryoturba- } \\
\text { tionen } \\
\text { Schwemm- } \\
\text { sand }\end{array}$ & $110-270$ & $\begin{array}{l}\text { Auelehm (Lößlehm) } \\
\text { Kryoturbationen } \\
\text { Schwemmsand } \\
\text { mit Frostspalten }\end{array}$ & \\
\hline
\end{tabular}




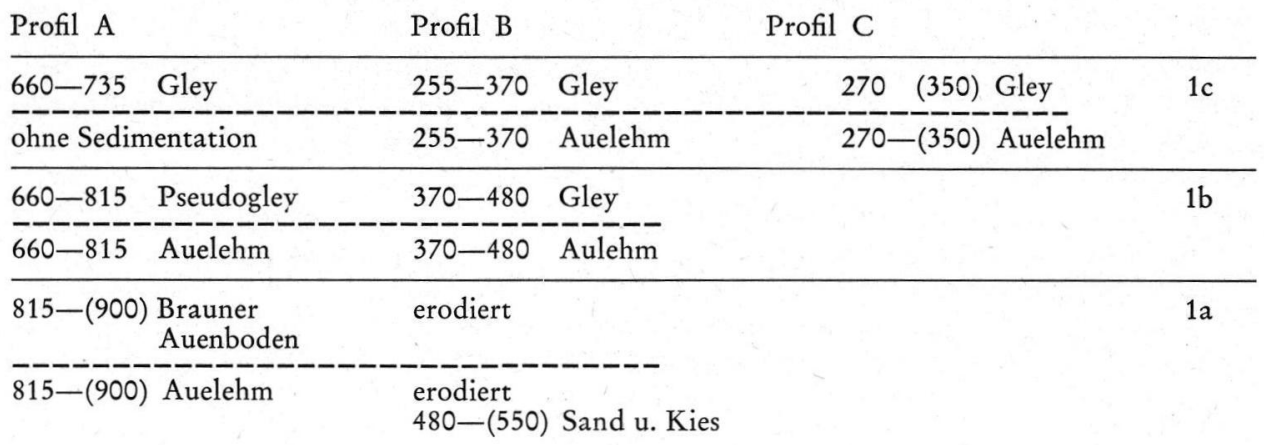

Das Profil kann in zwei Hauptabschnitte zerlegt werden, einen tieferen mit Auelehm (Nr. 1) und einen höheren mit Lößlehm (Nr. 2-5):

1a. Bildung eines Auenbodens aus Sedimenten der Hauptterrasse; z. T. (anschließend) geringfügige Ausräumung der Terrassensedimente.

1b. Füllung der ausgeräumten Zone mit Auelehm, seitlich über den Auenboden hinweggreifend. Abschließend entstand am Rande der Reliefdepression ein Pseudogley, der noch bis in den Auenboden hinunter wirkte, sonst ein Gley. Frostblätterigkeit spricht für winterliche Frostwirkung, wie sie z. B. auch ausgehende Abschnitte jüngerer Interglaziale kennzeichnet.

1c. Einer zweiten Füllungsphase folgt wiederum Gleybildung. Diese griff randlich über das Tälchen etwas hinaus, so daß der So-Horizont des unter $1 \mathrm{~b}$ erwähnten Pseudogleyes dadurch ebenfalls überformt wurde. Der Grad der Vergleyung entspricht etwa demjenigen, welcher in relativ stark ausgeprägten würmeiszeitlichen Tundragleyen des Alpenvorlandes vorkommt.

2. Ablagerung eines kiesig-sandigen Schwemmschicht; zugleich Bildung von Frostspalten. Anschließend Überdeckung mit Auelehm, anfänglich kryoturbate Verwürgungen mit der Schwemmschicht; synsedimentäre Vergleyung läßt nach dem Hangenden hin nach. Möglicherweise ist im Auelehm eine Lößkomponente enthalten; denn der Lößlehmcharakter tritt gegen den einstigen Talrand hin zunehmend deutlicher hervor. Im Talgrund bildete sich abschließend ein Naßgley, der seitlich in einen Pseudogley übergeht.

3. Ablagerung vom Staublehm; in der Reliefdepression vielleicht mit einer gewissen Neigung zur Solifluktion. Im Bereich dieser zunehmend ausgeglicheneren Geländevertiefung kam es abschließend wiederum zur Gleybildung; seitlich angrenzend entstand ein Pseudogley und noch weiter entfernt eine Pseudogley-Parabraunerde. Auch der Pseudogley führt in seinem Oberboden Merkmale einer späteren durch randlich weiter ausgreifendes Grundwasser bedingten Vergleyung.

4. Über die gesamte Profilstrecke wurde ein Lößlehm vom Typ des Fleckenlehms gelegt, dem anfänglich noch ein höherer Anteil an Fremdmaterial eingemischt wurde. Abschließend hat sich eine Parabraunerde gebildet.

5. Die oberste periglaziale Deckschicht besteht aus kiesigen Sanden und Lößlehm. Daraus ist der heutige Boden entstanden.

Die Abschnitte 2 bis 5 gehören in die 4 letzten Kaltzeiten. Getrennt werden sie durch interglaziale Böden und abgeschlossen durch den heutigen Boden. Der Abschnitt 1 leitet zum Komplex der Riesenböden über. Möglicherweise gehören $\mathrm{Nr}$. 1b und $1 \mathrm{c}$ noch in den oberen Abschnitt in die viertletzte Kaltzeit. Eine weitergehende Aufgliederung ist vorläufig nicht zweckmäßig.

Es würde an dieser Stelle zu weit führen, die Erforschungsgeschichte der Rheinterrassen noch einmal zu wiederholen. Jedenfalls scheint seit der Arbeit von H. W. Quitzow 
(1956) die Verknüpfung der jüngeren Terrassen des Mittelrheins mit denen des Niederrheins gesichert. Bis zu einem gewissen Grad gilt dies auch für die zeitliche Einstufung. Hinsichtlich der älteren Terrassen zeichnet sich dagegen eine laufende Zurückstufung ab, z. B.:

Kh. KAISER (1956): Hauptterrasse

Kh. KAISER (1957): Jüngere Hauptterrasse

$=$ Mindel

Kh. KAISER (1961): Jüngere Hauptterrasse

$=$ Günz und Frühmindel

$=$ Donaukaltzeiten und Günz

Nach den Untersuchungen von Kh. KaIser (1956, 1957), L. Ahorner (1962) und L. Ahorner \& Kh. Kaiser (1964) steht immerhin die kaltklimatische Natur der Hauptterrassen oder wenigstens deren Hauptglieder fest, und eine Zuordnung zum mittleren und älteren Großabschnitt des Quartärs ist aufgrund der Deckschichten, wie sie in Wegberg vorliegen, sicher.

f. Einstufung der Terrasen

Gemäß obiger Ausführungen ist eine zwanglose Eingliederung der die Deckschichten unterlagernden Terrassen in das pedostratigraphische Schema möglich, das in Süddeutschland (K. BRUNNACKER 1965) zumindest gebietsweise brauchbar ist (von unten nach oben):

3. Jüngerer Großabschnitt: Drei Interglaziale mit Parabraunerden zwischen den Eiszeiten, die im Alpenvorland als Günz, Mindel, Riß und Würm bezeichnet werden. Am Niederrhein: jüngere Obere Mittelterrasse, darin bereits Halsbandlemming (Fl. Heller \& K. Brunnacker 1966) und Frostspalten sowie Kryoturbationen, Untere Mittelterrasse, Krefelder Mittelterrasse, Niederterrasse.

2. Mittlerer Großabschnitt: Zeit der großen Talverschüttung mit Riesenböden = Mosbachium i. S. von K. D. Adam (1964). Am Niederrhein: Jüngere Hauptterrasse und anscheinend ältere Obere Mittelterrasse.

1. Alterer Großabschnitt: Reliktbodenzeit; mit den Donaukaltzeiten; vorläufig jedoch ohne genauere Fixierung = Villafranchium i. S. von K. D. AdAM (1964). Am Niederrhein: Altere Hauptterrasse.

S chrift tum :

Adam, K. D.: Die Großgliederung des Pleistozäns in Mitteleuropa. Stuttgarter Beiträge zur Naturkunde, Nr. 132, Stuttgart 1964.

Ahorner, L.: Untersuchungen zur quartären Bruchtektonik der Niederrheinischen Bucht. Eiszeitalt. u. Gegenw., 13, 24-105, Öhringen 1962.

Ahorner, L. \& KaISER, Kh.: Uber altpleistozäne Kalt-Klima-Zeugen (Bodenfrosterscheinungen) in der Niederrheinischen Bucht. Decheniana, 116, 3-19, Bonn 1964.

Brunnacker, K.: Böden des älteren Pleistozäns bei Regensburg. Geologica Bavarica, 53, 148-160, München 1964 (1964a). - - Über Ablauf und Altersstellung altquartärer Verschüttungen im Maintal und nächst dem Donautal bei Regensburg. Eiszeitalt. u. Gegenw., 15, 72-80, Ơhringen 1964 (1964b). - - Schätzungen über die Dauer des Quartärs, insbesondere auf der Grundlage seiner Paläoböden. Geol. Rdsch., 54, 415-428, Stuttgart 1965. - - Das Profil „Westwand“ der Ziegeleigrube Dreesen in Rheindahlen. Bonner Jb., 166, 344356, Bonn 1966.

Fink, J.: Die Subkommission für Lößstratigraphie der Internationalen Quartärvereinigung. Eiszeitalt. u. Gegenw., 16, 264-275, OOhringen 1965.

Frechen, J. \& Rosauer, E. A.: Aufbau und Gliederung des Würm-Löß-Profils von Kärlich im Neuwieder-Becken. Fortschr. Geol. Rheinld. Westf., 4, 225-265, Krefeld 1959.

Heller, Fl. \& BrunNACKer, K.: Halsbandlemming-Reste in einer Oberen Mittelterrasse des Rheins bei Niederaußem. Eiszeitalt. u. Gegenw., 17, 17-112, Öhringen 1966.

KAISER, Kh.: Geologische Untersuchungen über die Hauptterrasse in der Niederrheinischen Bucht. Sonderveröff. Geolog. Inst. Univ. Köln, 1, Köln 1956. - - Die Höhenterrassen der Bergischen Randhöhen und die Eisrandbildungen an der Ruhr. Sonderveröff. Geolog. Inst. Univ. Köln, 2, Köln 1957. - - Gliederung und Formenschatz des Pliozäns und Quartärs am Mittel- und Niederrhein, sowie in den angrenzenden Niederlanden unter besonderer Berücksichtigung der Rheinterrassen. Köln und die Rheinlande, Festschr. 33. Dtsch. Geogr. Tag. 1961 in Köln, 236-278, Wiesbaden 1961. 
KempF, E.: Das Holstein-Interglazial von Tönisberg im Rahmen des niederrheinischen Pleistozäns. Eiszeitalt. u. Gegenw., 17, 5-60, Ohringen 1966.

Mückenhausen, E.: Fossile Böden im nördlichen Rheinland. Z. Pflanzenern., Düngung, Bodenkd., 65, 81-103, Weinheim 1954. - - Über die Geschichte der Böden. Geol. Jb., 69, 501-516, Hannover 1955. - - Die stratigraphische Gliederung des Löß-Komplexes von Kärlich im Neuwieder Becken. Fortschr. Geol. Rheinld. West., 4, 283-300, Krefeld 1959.

Müller, E. H.: Art und Herkunft des Lösses und Bodenbildungen in den äolischen Ablagerungen Nordrhein-Westfalens unter Berücksichtigung der Nachbargebiete. Fortschr. Geol. Rheinld. West., 4, 247-254, Krefeld 1959.

PAAs, W.: Rezente und fossile Böden auf niederrheinischen Terrassen und deren Deckschichten. Eiszeitalt. u. Gegenw., 12, 165-230, Öhringen 1961.

Quirzow, W.: Die Terrassengliederung im Niederrheinischen Tiefland. Geol. en Mijnb., N. S. 18, 257-373, s'Gravenhage 1956.

Remy, H.: Die zeitliche Stellung der Rodderbergtuffe im rheinischen Löß. Decheniana, 112, 271278, Bonn 1959. - - Der Löß am unteren Mittel- und Niederrhein. Eiszeitalt. u. Gegenw., 11, 107-120, Ohringen 1960. - - Die Terrassen der vorletzten Vereisung am Rhein und an der Donau. Eiszeitalt. u. Gegenw., 14, 142-152, Ohringen 1963.

Remy, H. \& PAas, W.: Die Lößprofile von Koblenz-Metternich und Moselweiß. Fortschr. Geol. Rheinld. West., 4, 331-336, Krefeld 1959.

Manuskr. eingeg. 8. 4. 1967.

Anschrift des Verf.: Prof. Dr. K. Brunnacker, Geol. Inst. Univ., 5 Köln, Zülpicherstraße 49. 